\title{
The Persian irrational performance beliefs inventory (iPBI-Persian): Translation, confirmatory factor analysis, and test-retest reliability, in Iranian athletes
}

\section{Mohammadbagher Nejati $^{1}$ (D) Alireza Farsi $^{1}$ (D) Ebrahim Moteshareie ${ }^{1}$ (D) Martin J. Turner ${ }^{2}$ (D)}

Accepted: 21 April 2021 / Published online: 30 April 2021

(C) The Author(s) 2021

\begin{abstract}
This paper reports the development and initial validation of the Persian language Irrational Performance Beliefs Inventory (iPBI-Persian). The original iPBI was developed to provide a validated measure of the four core irrational beliefs of rational emotive behavior therapy (REBT) within performance-based samples, such as athletes. Data retrieved from 334 athletes (169 men, 165 women, Mage $=21.52 \pm 4.00$ years) were analyses using SPSS and LISREL software packages. After the linguistic and cross-cultural adaptation processes, confirmatory factor analysis (CFA) results showed that six items did not have acceptable factor loadings. After removal of problem items, a 22-item version was developed $(\mathrm{CFI}=0.96)$. The iPBI-Persian demonstrated excellent levels of reliability, with internal consistency and test-retest reliability, as well as construct validity. This paper indicates that the 22-item iPBI-Persian can be used as a self-assessment instrument to evaluate irrational performance beliefs in Iranian athlete samples.
\end{abstract}

Keywords CBT · Questionnaire · Applied · Measurement $\cdot$ Rational beliefs

Martin J. Turner

m.turner@mmu.ac.uk

Mohammadbagher Nejati

mohamadbaghernejati@gmail.com

Alireza Farsi

ar.farsi@gmail.com

Ebrahim Moteshareie

motesharee@ut.ac.ir

1 Faculty of Sport Sciences and Health, Shahid Beheshti University, Tehran, Iran

2 Department of Psychology, Faculty of Health, Psychology and Social Care, Manchester Metropolitan University, Manchester, UK 
Rational emotive behavior therapy (REBT) holds that, in response to adverse events, it is not the event itself that leads to dysfunctional emotions, but individual beliefs about the event that underpins these emotions (Ellis, 1957). Irrational beliefs underpin a range of dysfunctional emotions and maladaptive behaviors (Szentagotai \& Jones, 2010), and research has shown that irrational beliefs are associated with poorer mental health in athletes (e.g., Turner et al., 2019a, b). Importantly, REBT provides a specific intervention through which irrational beliefs are assessed and then disputed (i.e., via cognitive restructuring), following which rational beliefs are endorsed and strengthened (see Ellis, 1994). In sport, irrational beliefs are identified as an important risk factor for anxiety (Turner \& Barker, 2013), low self-acceptance (Cunningham \& Turner, 2016), fatigue (Turner \& Moore, 2016), low resilience (Deen et al., 2017), depression (Chotpitayasunondh \& Turner, 2019; Turner et al., 2019a, b), and poor athletic performance (Mesagno et al., 2020; Wood et al., 2017). A recent systematic review of the research concerning REBT with athletes indicates that REBT is especially useful in helping athletes to reduce anxiety (Jordana et al., 2020).

In order to advance research into the irrational beliefs of athletes, it is essential that researchers establish credible and reliable psychometrics for irrational beliefs (Terjesen et al., 2009). Research examining irrational beliefs in athletes has been limited historically by a lack of domain-specific psychometrics of irrational beliefs (Turner \& Barker, 2014). Recent recommendations state that the new criteria should take into account the conditions in the assessment of psychological structures (Ziegler \& Horstmann, 2015); therefore, the irrational Performance Beliefs Inventory (iPBI; Turner, Allen, et al., 2018; Turner, Slater, et al., 2018) was developed and validated with athletes (Turner \& Allen, 2018) as the first measure of irrational beliefs in performance domains such as sports, education, business, and the military. The iPBI has been used widely in REBT research involving athlete samples (Jordana et al., 2020). The iPBI has 28-items, comprising seven items for each of the four core irrational beliefs (e.g., Dryden \& Branch, 2008). The four core irrational beliefs are primary irrational beliefs (PIB), and three secondary irrational beliefs, namely awfulizing (AWF), low frustration tolerance (LFT), and depreciation (DEP). In the iPBI, and in line with contemporary REBT theory, the four core irrational beliefs are assessed in relation to ten performance content areas (including success, failure, approval, for example). Previous research has shown that the iPBI demonstrates construct, concurrent, and predictive validity (Turner et al. 2018a, b), test-retest reliability (Turner et al., 2018a), and is sensitive to change via REBT intervention (e.g., Deen et al., 2017).

Amidst the proliferation of REBT across various countries within the sports domain (Jordana et al., 2020), the iPBI has been translated and validated in languages other than English such as German (Chrysidis et al., 2020), Thai (Chotpitayasunondh \& Turner, 2019), and Turkish (Urfa \& Aşç1, 2018). This work is important because it allows irrational beliefs to be assessed more accurately (i.e., in line with contemporary theory) within the respective countries, and for the effectiveness of REBT to be monitored more rigorously, cross-culturally (Lega \& Ellis, 2001). To extend this work, the present paper adapts the iPBI into Persian and tests its validity and reliability. In Iran and other Persian-speaking countries, very few domestic 
research studies have reported the use of REBT with athletes, and to date there is no valid questionnaire to measure the irrational beliefs of Iranian-speaking athletes. Mostly, surveys aligned to REBT reflect general irrational beliefs rather than performance beliefs, such as the 100-item Jones Irrational Beliefs Questionnaire (IBQ; MozafariZadeh et al., 2018). The IBQ questionnaire has limitations that prevent its use in new and performance-related research. For example, many IBQ items measure emotions, rather than beliefs. Some items used to evaluate beliefs are actually tapping inferences or automatic thoughts, and the IBQ does not clearly align with contemporary REBT theory. It is clear that for the effects of REBT and the impact of irrational beliefs to be determined and recognized in Iranian athletes, there is a need to accurately measure irrational beliefs in Persian language. The Persian version of iPBI can help researchers to investigate the health consequences of irrational beliefs in Persian-speaking athlete samples, and provides a valid psychometric for practical work using REBT within Persian-speaking populations.

In order to prevent misinterpretations that occur when using psychological questionnaires, measures must be valid and reliable (Durand-Bush et al., 2001). The most important part in determining the validity of questionnaires within different cultural backgrounds is determining the construct validity of the questionnaire using confirmatory factor analysis (CFA; Brown, 2006). In addition, determining validity and reliability is an important requirement and presupposition of psychological measurement, which is related to the repeatability of answers in different situations and times. Thus, it is necessary to examine the temporal reliability and internal consistency of questionnaires via test-retest reliability analyses. Therefore, our aim in this paper is to investigate the construct validity (factor) and reliability of Persian version of the iPBI (iPBI-Persian) among male and female Persian-speaking athletes. To meet these aims, the original iPBI was translated into Persian language from the original English for the first time in research, and the iPBI-Persian was tested for validity and reliability in Iranian athletes.

\section{Method}

\section{Participants}

Participants included elite, skilled and semi-skilled (female $=182$, male $=238)$ athletes across ten sports including team disciplines such as: football $(n=81)$, handball $(n=24)$, volleyball $(n=69)$ and individual disciplines such as: badminton $(n=45)$, table tennis $(n=32)$, swimming $(n=40)$, track and field $(n=29)$, karate and taekwondo $(n=36)$ in Iran. Considering that the sample required in factor analysis studies has been proposed to be 10 to 15 participants per item (Kline, 2015; Meyers et al., 2016), initially 420 questionnaires were distributed among Iranian athletes. Data for 42 participants were discarded due to systematic answers, distorted demographic information, or failure to answer a significant number of questions. Data for a further 44 participants were excluded from the analyses due to univariate outlier scores (z scores higher than 2.5 or less than -2.5) or multivariate Mahalanobis distance $p<0.001$; see (Tabachnick \& Fidell, 2001). As a result, the final analyses 
were performed on the data of 334 athletes (female $=165$, males $=169$ ) with an age range of 14 to 35 years $(M=18.76, S D=3.12)$ at three skill levels: elite $(n=35)$, skilled $(n=202)$, beginner $(n=97)$, across team $(n=158)$ and individual $(n=176)$ sports. Myers et al. (2011) reported that a sample size of more than 300 participants is good for factor analysis research. It is noteworthy that the purpose of selecting this wide range in the level of skill and type of sport is to increase the generalizability of research findings (Terry et al., 2003).

\section{Measures}

The iPBI (Turner et al. 2018a, b) is a 28-item self-report scale that measures the four core irrational beliefs of REBT; PIB (e.g., "I have to be respected by the members of my team"), LFT (e.g.: "I can't stand not reaching my goals"), AWF (e.g., "It's awful to not be treated fairly by my peers"), and DEP (e.g., "If I face setbacks it goes to show how stupid I am"). Responses are made on a five-point scale from 1 (strongly disagree) to 5 (strongly agree). The iPBI represents construct and concurrent validity in organizational and sport contexts (Turner \& Moore, 2016) and offers strong fit index $(\mathrm{CFI}=0.93, \mathrm{NNFI}=0.92, \mathrm{SRMR}=0.06$, RMSEA $=0.07$; Turner et al. 2018a, b). Scores on the iPBI also correlate with scores on similar subscales of a corresponding measure of irrational beliefs - the shortened general attitude and belief scale (Lindner et al., 1999). The iPBI was developed for achievement contexts (Turner et al. 2018a, b) and is used frequently to measure irrational beliefs in athletic samples (Jordana et al., 2020).

For the current study, the original English language iPBI was translated into Persian (iPBI-Persian) and then participants were asked to rate their agreement with the 28-items on a 5-point Likert scale from 1 (strongly disagree) to 5 (strongly agree). Higher scores indicate greater irrational beliefs. The original 28-item iPBI shows good internal consistency $(\alpha=0.90-0.96)$ and criterion validity $(r=0.47-0.81)$ in the professional working environment (Turner et al. 2018a, b), and in athlete samples the 20 -item iPBI-2 shows good internal consistency $(\alpha=0.79-0.87)$ and acceptable fit indices (Turner \& Allen, 2018), and has shown good test-retest reliability in samples of college students and athletes (Turner et al. 2018a, b).

\section{Procedure}

Ethical approval was received from the University Research Ethics Committee prior to all data collection. The original English iPBI was translated into Persian using the translation back-translation method (Brislin, 1986; Flaherty et al., 1988). This method is widely used and is the standard method for translating research tools from one language to another (Kvamme et al., 1998). The first stage-translationrequired the presence of two experts with complete mastery of both languages (English-Persian) to translate the tool from English to Persian. The second step was the approval of the translation by a group of two fluent English-speaking sports psychologists to convey the full meaning of the original version. The third step was the back translation of the items into English by another bilingual (English-Persian) 
expert who did not have access to the original version. The final step was the evaluation of the translation by a committee that approved the translations. Any differences between versions were checked and changed so that the translated version matched the original version exactly.

An online questionnaire link containing the iPBI-Persian was sent to the personal emails or social media accounts of participating athletes. Participants received information about the research and their participation was anonymous, and the data was kept confidential. The respondents were assured that their responses would be confidential and would only be used for research purposes. In addition, to reduce social desirability among respondents, participants were informed that the results of the study had no effect on their selection in the relevant sports and that there were no right or wrong answers to the questions (Terry et al., 2003). All participants provided informed consent prior to completing the questionnaire.

\section{Data analysis}

According to structural equation experts, when researchers have an assumed theoretical model related to data structure, the statistical method used in the first step should be confirmatory factor analysis (CFA) rather than exploratory (Schutz et al., 1993). Therefore, we applied CFA to examine the construct (factor) validity of the of the iPBI-Persian (Brown, 2006). The internal consistency of the iPBI-Persian was determined using Cronbach's alpha coefficients. In order to evaluate the consistency of the results obtained from the iPBI-Persian, a value of 0.70 or higher was considered acceptable (Nunnally, 1994; Terwee et al., 2007). For tests of stability and the reproducibility of the measure, test-retest reliability over a two-week interval was examined by calculating interclass correlation coefficients (ICC). According to Merletti et al., (1998), values of 0.80 to 1.00 are excellent for the ICC, 0.60 to 0.80 are good, and values below 0.60 are unacceptable.

In CFA and in relation to the report of fitness indicators, considering that there is no general agreement among structural equation modeling experts on which one of the fitness indicators provides a better estimate of the model, it is recommended to report a combination of three to four indices (Kline, 2015). Also, considering that the fitness indicators are placed in three groups: absolute, comparative, and parsimonious, and the indicators of each class provide different information about the fitness of the model, it is suggested that at least one indicator from each class to be reviewed and reported (Browne \& Cudeck, 1993). For fit indices, different parameters have been proposed by experts. In the present study, among the absolute fitness indices, $\chi 2 / \mathrm{df}$, RMSEA and SRMR, among the adaptive or comparative fit index, Tucker-Lewis Index (TLI), or Bentler Bonnet Non-Normed Fit Index (NNFI), as well as the Comparative Fitness Index (CFI), and finally the Parsimony Goodness of Fit Index (PGFI) and the Parsimony Normed of Fit Index (PNFI) were used. In the TLI and CFI index whose range of variation is between zero and one, values greater than 0.90 indicate good fit of the model, and values higher than 0.95 indicate very good fit of the model (Hu \& Bentler, 1999). For the RMSEA index, values less than 0.08 indicate an acceptable model and less than 0.06 indicate a good model $(\mathrm{Hu} \&$ 
Bentler, 1999). Also, for this index, a confidence interval can be calculated, and ideally, the lower limit of the confidence interval is close to zero and the upper limit is not more than 0.1 . For the SRMR index, values less than 0.08 are acceptable $(\mathrm{Hu}$ $\&$ Bentler, 1999). There is no general agreement on acceptable values for the Chisquare-to-DF index, with some researchers considering values less than three to be acceptable and good (Kline, 2015) and others consider values two to five as a sign of reasonability and appropriateness of model (Terry et al., 2003).

Finally, we used Multivariate analysis of variance (MANOVA) to compare subscales of iPBI-Persian scores between skill levels, and gender. To perform the above statistical calculations, SPSS 20 and LISREL 8.8 (Feldt et al., 2007) were used.

\section{Results}

All means, standard deviation and Pearson correlation coefficients were calculated for all variables shown in Table 1 .

\section{Confirmatory Factor Analysis}

Prior to the first-order CFA of the iPBI-Persian questionnaire, the univariate and multivariate normality of the data was measured by LISREL and examined through the standardized value of skewness and kurtosis indices (value divided by standard error between \pm 1.96 ) for univariate normality and Mardia coefficient (Mardia, 1970) for multivariate normality (Tabachnick \& Fidel, 2001). The results showed that univariate normality was not present in 21 items (standardized value of skewness and kurtosis greater than 1.96 or less than negative 1.96) and the non-significance of Mardia index in multivariate normality (Mardia's Kurtosis $=85,973.85, p<0.001$ ) in the present study. Since the maximum likelihood (ML) estimation method is sensitive to the non-normal distribution of data and leads to underestimation of adaptive fit index and overestimation of chi-square index, therefore, to perform CFA, the

Table 1 Means, SDs, and intercorrelation of variables

\begin{tabular}{lrllllllll}
\hline Factor & $M$ & SD & Total-iPBI & PIB & LFT & AWF & DEP & Skewness & Kurtosis \\
\hline Total iPBI & 2.9 & 0.56 & - & $0.54 * *$ & $0.73 *$ & $0.81^{* *}$ & $.70^{* *}$ & .2 & -.20 \\
PIB & 2.9 & 0.78 & $0.54 * *$ & - & $0.22 *$ & $0.22^{* *}$ & $0.17 * *$ & 0.078 & -0.20 \\
LFT & 3.3 & 0.89 & $0.73^{* *}$ & $0.22^{* *}$ & - & $0.57^{* *}$ & $0.33^{* *}$ & -0.22 & -0.46 \\
AWF & 30.3 & 0.77 & $0.81^{* *}$ & $0.22^{* *}$ & 0.57 & - & $0.40^{* *}$ & -0.17 & -0.27 \\
DEP & 2.8 & 0.77 & $0.70^{* *}$ & $0.17 * *$ & 0.33 & $0.40^{* *}$ & - & 0.71 & -0.08 \\
\hline
\end{tabular}

$N=166$

iPBI-Persian version of Irrational Performance Beliefs Inventory, PIB personal irrational beliefs, $L F T$ low frustration tolerance, AWF awfulizing, DEP depreciation,

Correlation between iPBI-Persian subscales and total scores from 22 items were computed with the items removed from the total score. Cronbach's alphas are shown in the diagonal

$* * p<0.01 ; * p<0.05$ 
robust maximum likelihood estimation method (RML) of Satura-Bentler was used (Mels, 2006).

The results of first-order CFA using the robust maximum likelihood (RML) estimation method indicate that some parameters of the model are not significant in the iPBI-Persian measurement model. In modeling structural equations, the statistical index is used to test the path coefficient (factor load) between the observed variables (questions) and the latent variables (factors) in the model, therefore, parameters with t values greater than 1.96 are statistically significant $(p<0.05)$. Otherwise, and if the path coefficient is not significant, that item must be removed to continue the analysis (Browne \& Cudeck, 1993).

A careful examination of the initial model shows that two PIB questions ("decisions that affect me must be justified", and "I must be liked by the people who are important to me"), three LFT items ("I cannot tolerate not getting the opportunity", "I cannot tolerate not achieving my goals", and "I cannot tolerate failure in cases that are important to me"), and one DEP item ("if my competencies do not grow and improve continuously, it would show what a failure I am"), had low t values, that were less than the acceptable value of 1.96. Also, the examination of internal consistency showed that all the mentioned questions would lead to a decrease in Cronbach's alpha coefficient and they were candidates for elimination. As a result, these six questions were deleted, and the modified model underwent CFA.

After removing the above six items, the results of CFA for the modified model (Fig. 1) shows a very good fit and all values and parameters of the model (Table 2) are significant $(p<0.001)$. The fit indices of the modified model are reported in Table 3.

As can be seen in Table 3, after removing the six items, all comparative fit indices were higher than 0.95 , RMSEA index with a value of 0.06 was lower than 0.08 and PGFI and PNFI with values of 0.70 and 0.82 above the acceptable cut-off point 0.6 , all of which indicate the acceptable and excellent fit of the first-order modified model. Thus, the results of the CFA showed that the iPBI-Persian after removing two items from PIB factor, three items from LFT factor and one item from DEP factor, had an acceptable and appropriate factor validity. In addition, parameters (non-standard factor load) and t-index on the relationship between questions with the relevant subscales, shows that the $t$ value in all items is higher than 1.96 , which indicates a significant relationship between questions and related factors. So that all observed variables (items) are able to predict their factors (Browne et al., 2010).

\section{Second-order factor analysis}

In order to achieve a more accurate factor structure, the second-order factor analysis method was used. In such models, it is assumed that the latent variables themselves in the common variance are due to one or more higher order factors. In other words, second-order factors are considered first-order factors. This method is used to study the appropriateness of the operating structure of the questionnaire and to confirm the existence of the constituent components or related research (Browne et al., 2010). 


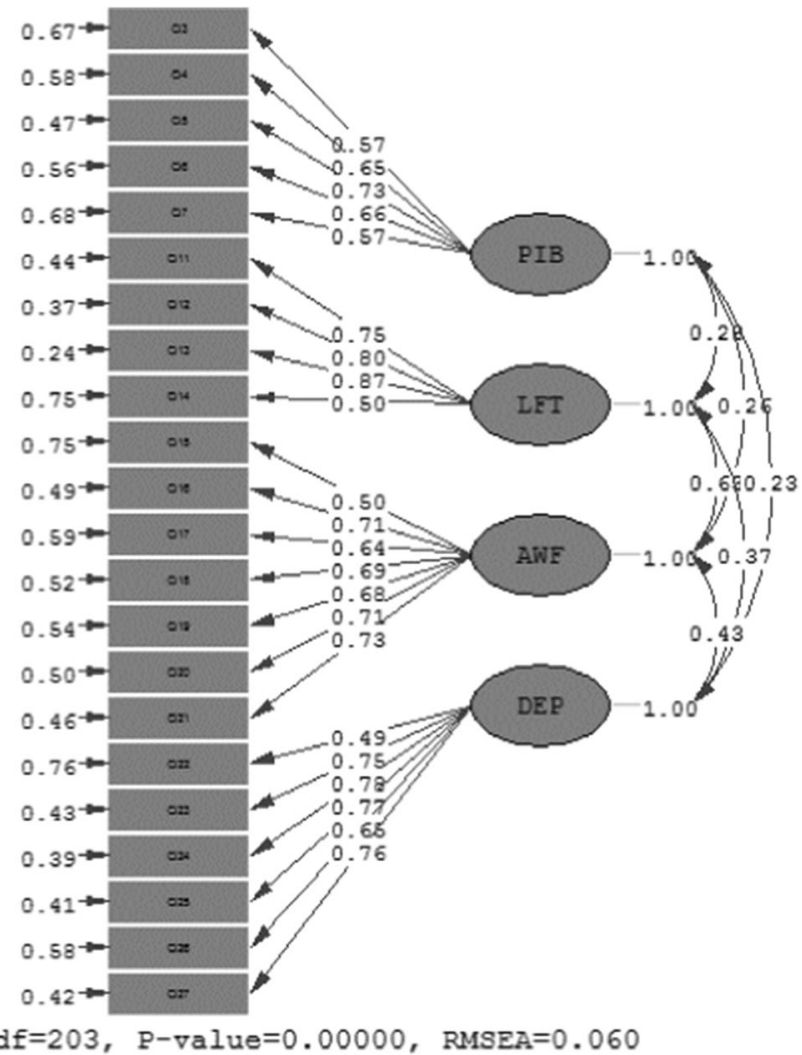

Fig. 1 Standard factors for 22 items of the four-factor iPBI-Persian model

The results of the second-order CFA of the modified model (Fig. 2) show a very good fit and all values and parameters of the model are significant $(p<0.001)$. The fit indices of the modified model are also reported in Table 4.

\section{Population-based differences}

The scores of all four iPBI-Persian subscales were compared across skill levels (beginner, skilled, and elite) and between participant gender. The result did not show any significant differences between skill levels, or gender, in any of the subscales $(p>0.05)$.

\section{Scale reliability}

The results of Cronbach's alpha coefficient are presented in Table 5 to determine the internal consistency of the subscales of the iPBI-Persian. Internal consistency of total iPBI-Persian (composite) with 22 items was 0.87 and the internal consistency of other subscales of the questionnaire was in the acceptable range $(\alpha>0.70)$. 


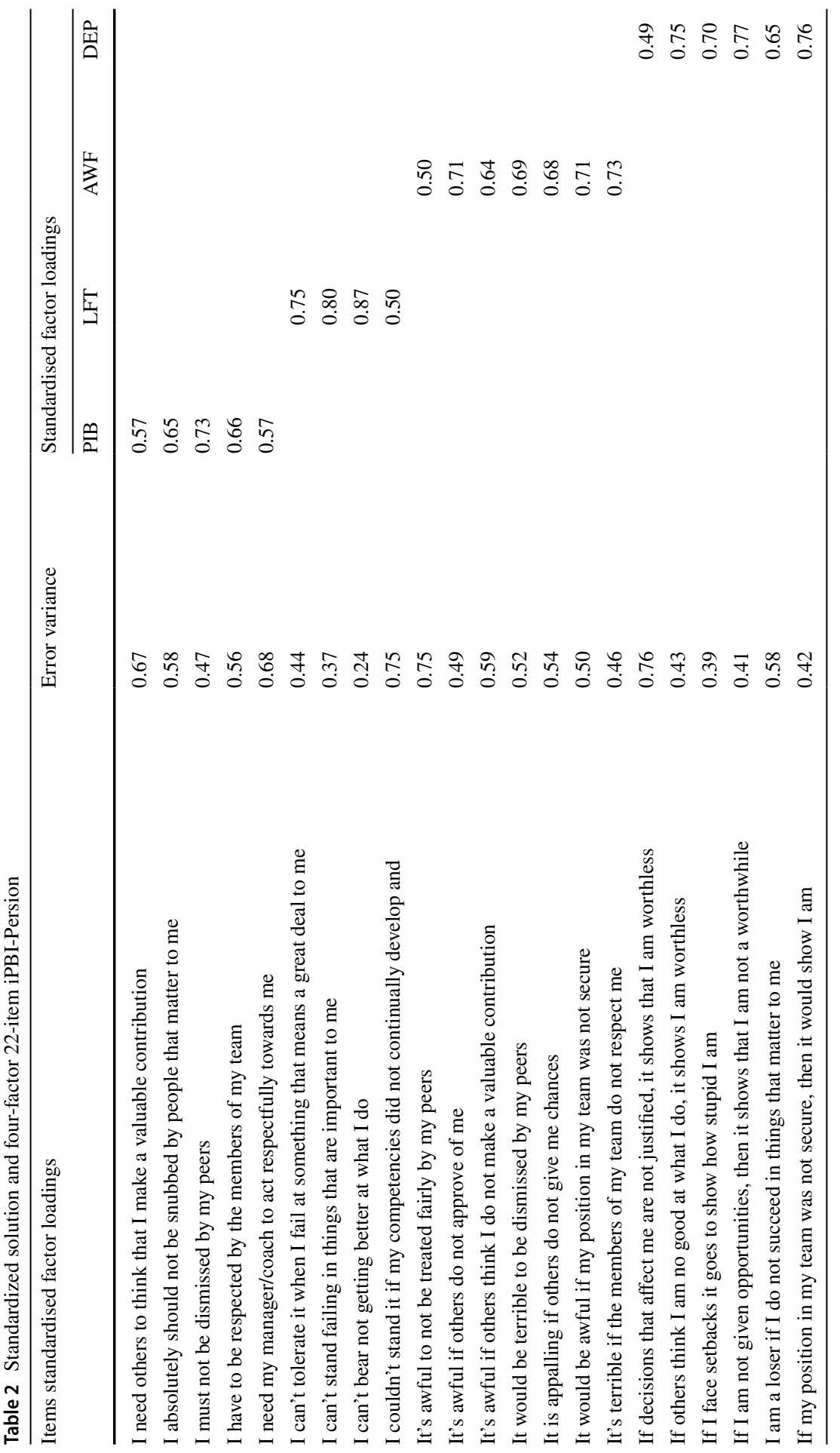


Table 3 Fitness indices of first order CFA of the modified iPBI-Persian questionnaire

\begin{tabular}{llllllll}
\hline fit indices & $\chi^{2} / \mathrm{DF}$ & CFI & TLI & SRMR & PGFI & PNFI & RMSEA \\
\hline Visible value & 2.23 & 0.96 & 0.96 & 0.06 & 0.70 & 0.82 & 0.06 \\
Acceptable values & $<3$ & $>0.90$ & $>0.90$ & $<0.06$ & $>0.6$ & $>0.6$ & $<0.08$ \\
\hline
\end{tabular}

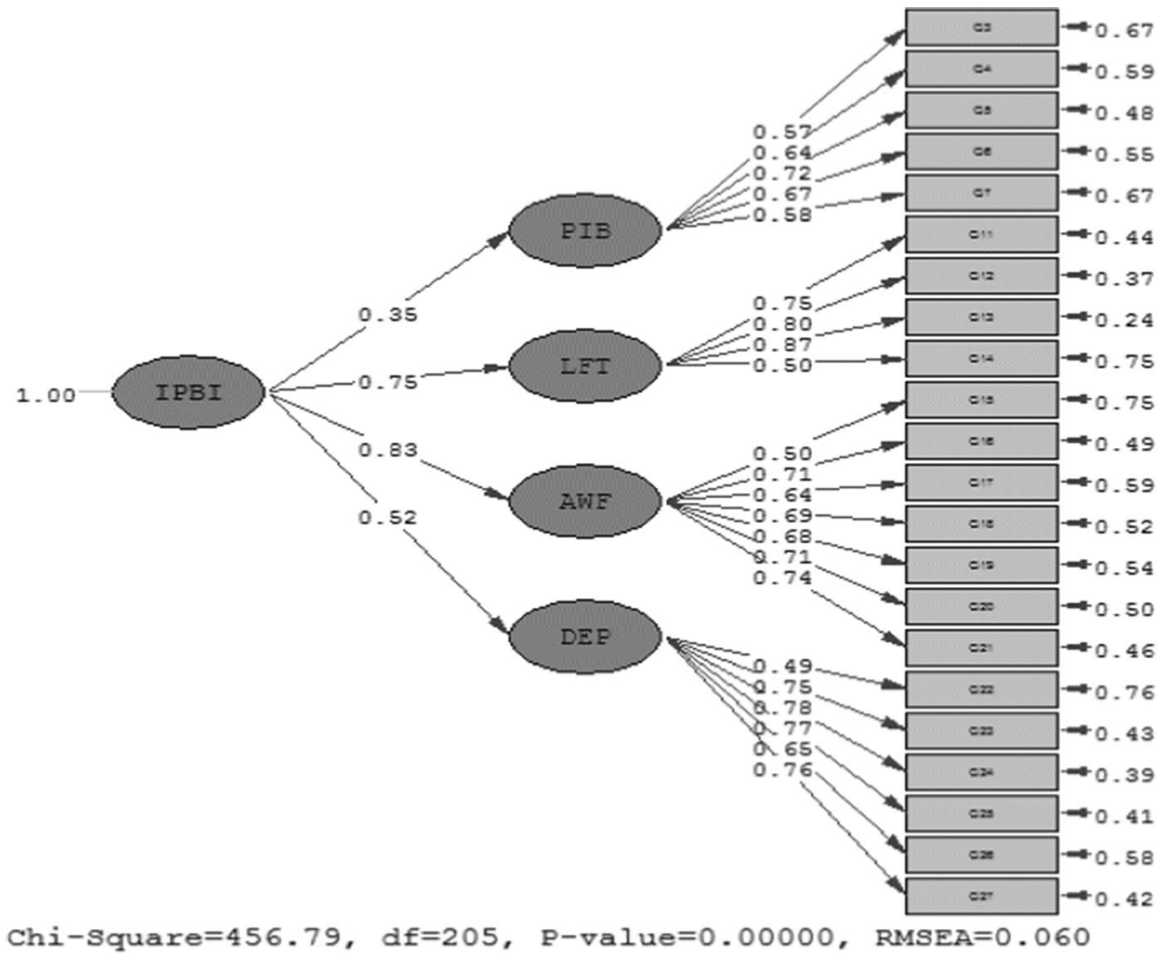

Fig. 2 Standard factor for the second row of 22 items of the four-factor iPBI-Persian model

Table 4 Fitness indices of confirmatory factor analysis- second order of the modified iPBI-Persian questionnaire

\begin{tabular}{llllllll}
\hline Fit indices & $\chi^{2} / \mathrm{DF}$ & CFI & TLI & SRMR & PGFI & PNFI & RMSEA \\
\hline Visible values & 2.22 & 0.96 & 0.96 & 0.06 & 0.71 & 0.83 & 0.06 \\
Acceptable values & $<3$ & $>0.95$ & $>0.95$ & $<0.06$ & $>0.6$ & $>0.6$ & $<0.08$ \\
\hline
\end{tabular}

Test-retest reliability shows the repeatability of a criterion and its ability to provide continuous scores over time in a fixed population (Enns et al., 2002). A valid reliable criterion for irrational beliefs should show that scores are constant over time and that scores are significantly reduced only if REBT is used (Turner et al., 2018a). 
Table 5 Results of Cronbach's alpha coefficient and intra-class correlation coefficient (ICC) to determine the internal stability and temporal reliability of the iPBI-Persian

\begin{tabular}{|c|c|c|}
\hline Subscales & Alpha coefficient & $\begin{array}{l}\text { Inter-class correlation } \\
\text { coefficient ICC }(95 \% \\
\text { CI })\end{array}$ \\
\hline PIB $(n=5)$ & 0.77 & $0.92(0.86$ to 0.95$)$ \\
\hline $\operatorname{LFT}(n=4)$ & 0.81 & $0.94(0.90$ TO 0.96$)$ \\
\hline $\mathrm{AWF}(\mathrm{n}=7)$ & 0.84 & $0.89(0.82$ TO 0.93 \\
\hline $\operatorname{DEP}(n=6)$ & 0.84 & $0.86(0.77$ TO 0.91$)$ \\
\hline TOTAL P-IPBI $(\mathrm{n}=22)$ & 0.87 & 0.96 (0.93 ТО 0.97) \\
\hline
\end{tabular}

This is important because many studies that use REBT in the field of sports have a single case design (Barker et al., 2011) in which the results are frequently evaluated in basic and intervention courses, therefore psychometrics should be reliable in repeated evaluations. According to the results of Table 5, the values of interclass correlation coefficients (ICC) of the subscales obtained with a two weeks interval ranged from 0.89 in AWF to 0.94 in LFT and the values of all subscales were higher than the acceptable cut point (0.75), which indicates the acceptability of temporal reliability or repeatability of subscales in the iPBI-Persian. Also, the value of the interclass correlation coefficients (ICC) total iPBI-Persian with an average of 0.96 and a range of 0.93 to 0.97 in the confidence interval of 0.95 , confirms the temporal reliability of the iPBI-Persian.

\section{Discussion}

In the present study we evaluated the construct validity and reliability of the Persian language irrational Performance Beliefs Inventory (iPBI-Persian) using CFA and the Robust Maximum Likelihood Estimation (RMLE) method. The results of factor analysis showed that the 28-item iPBI-Persian did not have a good fit. A closer inspection of the model showed that the factor loadings of six items had low t values, and thus, were removed prior to a second CFA. The CFA model for the 22-item iPBI-Persian showed that all fitness indices had excellent and appropriate values, which indicated the modified model was a good fit to the data.

Comparison of the results of the present study with the results of the original iPBI (English language) shows some differences. In the study by Turner et al. (2018a, b), all 28-items sufficiently loaded onto the appropriate and intended factors, but for the iPBI-Persian this was not the case and therefore item removal took place prior to a second CFA. The inconsistency between the iPBI-Persian and the original iPBI can be attributed to the community and the sample used in the research. Simply put, the sample used in Turner et al. (2018a) was an occupational sample based in the United Kingdom, whilst the validity and reliability of the iPBI-Persian has been measured in the athlete community in Iran, and as such, phrases related to job and work environment were omitted. Further, differences can be attributed to cultural differences 
and differences in respondents' perceptions. The phrases used in the questions "I cannot stand the failure in achieving my goals" and "I cannot stand to fail in important tasks" are repetitive that can be attributed to the translation process and that these phrases convey the same concept in Persian language. In other words, different CFA results between the iPBI-Persian and the original English iPBI can be attributed to cultural differences that have been improperly understood or interpreted or ignored by Iranian athletes.

The results of Turner and Allen (2018), who measured the validity and reliability of IPBI in the semi-professional and amateur athlete samples, are very consistent with the present study. In Turner and Allen (2018) eight questions that did not have the appropriate factor loading and were omitted, however, four factors were extracted and confirmed. The results of the research by Chotpitayasunondh and Turner (2019), in which a Thai language iPBI was validated, are in line with the current paper too, because eight items were omitted, and a number of omitted items are consistent with those omitted in the iPBI-Persian. However, in another study that examined the Psychometric Properties of the Turkish iPBI-2 (20 items; Urfa \& Asci, 2018), results confirmed the validity and reliability of the 20-item, 4-factor structure of iPBI-2, which is not entirely consistent with our results. In other words, in our results six items from the original iPBI; were omitted, with only two items in common with the eight omitted to form the iPBI-2 (Turner \& Allen, 2018).

Concerning the reliability of the IPBI-Persian in the present study, the results demonstrated internal stability, which is in line with the results of Turner and Allen (2018) and Chotpitayasunondh and Turner (2019). Regarding test-retest reliability of iPBI-Persian, results with a two-week interval indicated temporal reliability or repeatability. The ability to retest not only showed that the results were stable, but also showed that irrational beliefs may not change in the short-term in the absence of an REBT intervention.

One of the limitations of the present study was that Persian-speaking athlete samples were limited to Iranian athletes. As a result, in future research, it is suggested that other Persian-speaking athletes (e.g., from Tajikistan, Afghanistan) be considered. Also, further studies should investigate the psychometric properties of the iPBI-Persian via different methods such as Rasch analysis, because determining the validity and reliability of questionnaires is an ongoing process. And psychometric instruments need to be up-to-date. These methods can be used in future research. (Andrich, 1988), Multi-trait Multi-method (MTMM; Campbell \& Fiske, 1959), and Item Response Theory (Hambleton \& Swaminathan, 1985).

The results of the current study show that the iPBI-Persian is a valid and reliable psychometric to examine irrational beliefs in Persian-speaking athletes. It is the first measurement tool to measure the irrational performance beliefs of Persian-speaking athletes. The adaptation of the scale will contribute to conducting further studies on irrational performance beliefs, helping researchers to identify irrational beliefs in athletes, and helping sport psychologists to measure irrational beliefs in Iranian athletes.

Acknowledgements We would like to thank all of the participants who took part in this study. 


\section{Declarations}

Conflict of interest The authors declare that the research was conducted in the absence of any commercial or financial relationships that could be construed as a potential conflict of interest.

Open Access This article is licensed under a Creative Commons Attribution 4.0 International License, which permits use, sharing, adaptation, distribution and reproduction in any medium or format, as long as you give appropriate credit to the original author(s) and the source, provide a link to the Creative Commons licence, and indicate if changes were made. The images or other third party material in this article are included in the article's Creative Commons licence, unless indicated otherwise in a credit line to the material. If material is not included in the article's Creative Commons licence and your intended use is not permitted by statutory regulation or exceeds the permitted use, you will need to obtain permission directly from the copyright holder. To view a copy of this licence, visit http://creativecommons.org/licen ses/by/4.0/.

\section{References}

Andrich, D. (1988). Rasch models for measurement. Thousand Oaks: Sage.

Barker, J., McCarthy, P., Jones, M., \& Moran, A. (2011). Single case research methods in sport and exercise. London: Routledge.

Brislin, R. W. (1986). The wording and translation of research instruments. In W. J. Lonner, J. W. Berry (Eds.), Cross-cultural research and methodology series, Vol. 8. Field methods in crosscultural research (pp. 137-164). Sage Publications Inc.

Brown, T. (2006). Confirmatory factor analysis for applied research. The Guilford Press.

Browne, M. W., \& Cudeck, R. (1993). Alternative ways of assessing model fit. In K. A. Bollen \& J. S. Long (Eds.), Testing structural equation models (pp. 136-162). Newbury Park, CA: Sage.

Browne, C. M., Dowd, E. T., \& Freeman, A. (2010). Rational and irrational beliefs and psychopathology. Rational and irrational beliefs: Research, theory, and clinical practice, 149-172

Campbell, D. T., \& Fiske, D. W. (1959). Convergent and discriminant validation by the multitraitmultimethod matrix. Psychological Bulletin, 56(2), 81

Chotpitayasunondh, V., \& Turner, M. (2019). The Development and Validation of the Thai-Translated Irrational Performance Beliefs Inventory (T-iPBI). Journal of Rational-Emotive \& CognitiveBehavior Therapy, 37(2), 202-221

Chrysidis, S., Turner, M. J., \& Wood, A. G. (2020). The effects of REBT on irrational beliefs, selfdetermined motivation, and self-efficacy in American Football. Journal of Sports Sciences, 38(19), 2215-2224

Cunningham, R., \& Turner, M. J. (2016). Using Rational Emotive Behavior Therapy (REBT) with Mixed Martial Arts (MMA) athletes to reduce irrational beliefs and increase unconditional selfacceptance. Journal of Rational-Emotive \& Cognitive-Behavior Therapy, 34(4), 289-309

Deen, S., Turner, M. J., \& Wong, R. S. (2017). The effects of REBT, and the use of credos, on irrational beliefs and resilience qualities in athletes. The Sport Psychologist, 31(3), 249-263

Dryden, W., \& Branch, R. (2008). Fundamentals of rational emotive behaviour therapy: A training handbook. New Jersey: John Wiley \& Sons.

Durand-Bush, N., Salmela, J. H., \& Green-Demers, I. (2001). The Ottawa mental skills assessment tool (OMSAT-3*). The Sport Psychologist, 15(1), 1-19

Ellis, A. (1957). Rational psychotherapy and individual psychology. Journal of Individual Psychology, 13(1), 38-44

Ellis, A. (1994). Reason and emotion in psychotherapy. Secaucus, NJ: Birch lane

Enns, M. W., Cox, B. J., \& Clara, I. (2002). Adaptive and maladaptive perfectionism: Developmental origins and association with depression proneness. Personality and Individual Differences, 33(6), 921-935 
Feldt, T., Lintula, H., Suominen, S., Koskenvuo, M., Vahtera, J., \& Kivimäki, M. (2007). Structural validity and temporal stability of the 13-item sense of coherence scale: Prospective evidence from the population-based HeSSup study. Quality of life research, 16(3), 483

Flaherty, J. A., Gaviria, F. M., Pathak, D., Mitchell, T., Wintrob, R., Richman, J. A., \& Birz, S. (1988). Developing instruments for cross-cultural psychiatric research. Journal of Nervous and Mental Disease, 176(5), 257-263

Hambleton, R. K., \& Swaminathan, H. (1985). A look at psychometrics in the Netherlands. Nederlands Tijdschrift voor de Psychologie, 40, 446-451

Hu, L. T., \& Bentler, P. M. (1999). Cutoff criteria for fit indexes in covariance structure analysis: Conventional criteria versus new alternatives. Structural equation modeling: a multidisciplinary journal, 6(1), 1-55

Jordana, A., Turner, M. J., Ramis, Y., \& Torregrossa, M. (2020). A systematic mapping review on the use of Rational Emotive Behavior Therapy (REBT) with athletes. International Review of Sport and Exercise Psychology. https://doi.org/10.1080/1750984X.2020.1836673

Kline, R. B. (2015). Principles and practice of structural equation modeling. New York: Guilford publication.

Kvamme, O., Mainz, J., Helin, A., Ribacke, M., Olesen, F., \& Hjortdahl, P. (1998). Interpretation of questionnaires. An translation method problem. Nordisk Medicin, 113(10), 363-366

Lega, L. I., \& Ellis, A. (2001). Rational Emotive Behavior Therapy (REBT) in the new millenium: A cross-cultural approach. Journal of rational-emotive and cognitive-behavior therapy, 19(4), 201-222

Lindner, H., Kirkby, R., Wertheim, E., \& Birch, P. (1999). A brief assessment of irrational thinking: The shortened general attitude and belief scale. Cognitive Therapy and Research, 23(6), 651-663

Mels, G. (2006). LISREL for Windows: Getting started guide. Scientific Software International.

Merletti, R., Fiorito, A., Lo Conte, L. R., \& Cisari, C. (1998). Repeatability of electrically evoked EMG signals in the human vastus medialis muscle. Muscle \& Nerve, 21(2), 184-193. https://doi.org/10. 1002/(sici)1097-4598(199802)21:2<184::aid-mus5>3.0.co;2-7

Mesagno, C., Tibbert, S. J., Buchanan, E., Harvey, J. T., \& Turner, M. J. (2020). Irrational beliefs and choking under pressure: A preliminary investigation. Journal of Applied Sport Psychology. https://doi.org/10.1080/10413200.2020.1737273

Meyers, L. S., Gamst, G., \& Guarino, A. J. (2016). Applied multivariate research: Design and interpretation. Thousand Oaks: Sage publications.

MozafariZadeh, M., GhalechehYazdani, S., Heidari, F., \& Shabani, M. (2018). Effect of Rational Emotive Behavioral therapy on irrational beliefs of semi-professional adolescents rock climbers. Applied Psychological Research Quarterly, 9(2), 89-104

Myers, N. D., Ahn, S., \& Jin, Y. (2011). Sample size and power estimates for a confirmatory factor analytic model in exercise and sport: A Monte Carlo approach. Research Quarterly for Exercise and Sport, 82(3), 412-423. https://doi.org/10.1080/02701367.2011.105997

Nunnally, J. C. (1994). Psychometric theory3E. New York: Tata McGraw-hill education.

Schutz, R. W., \& Gessaroli, M. E. (1993). Use, misuse, and disuse of psychometrics in sport psychology research. In R. N. Singer, M. Murphey, \& L. K. Tennant (Eds.), Handbook of research on sport psychology (pp. 901-917). New York: Macmillan.

Szentagotai, A., \& Jones, J. (2010). The behavioral consequences of irrational beliefs. In D. David, S. J. Lynn, \& A. Ellis (Eds.), Rational and irrational beliefs: Research, theory, and clinical practice. (pp. 75-97). Oxford: Oxford University Press.

Tabachnick, G. B., \& Fidell, L. S. (2001). Using multivariate statistics (4th ed.). Needham Heights, MA: Allyn \& Bacon

Terjesen, M. D., Salhany, J., \& Sciutto, M. J. (2009). A psychometric review of measures of irrational beliefs: Implications for psychotherapy. Journal of Rational-Emotive \& Cognitive-Behavior Therapy, 27(2), 83-96

Terry, P. C., Lane, A. M., \& Fogarty, G. J. (2003). Construct validity of the Profile of Mood StatesAdolescents for use with adults. Psychology of sport and exercise, 4(2), 125-139

Terwee, C. B., Bot, S. D., de Boer, M. R., van der Windt, D. A., Knol, D. L., Dekker, J., et al. (2007). Quality criteria were proposed for measurement properties of health status questionnaires. Journal of Clinical Epidemiology, 60(1), 34-42

Turner, M., \& Allen, M. S. (2018). Confirmatory factor analysis of the irrational Performance Beliefs Inventory (iPBI) in a sample of amateur and semi-professional athletes. Psychology of Sport and Exercise, 35, 126-130 
Turner, M., Aspin, G., \& Gillman, J. (2019a). Maladaptive schemas as a potential mechanism through which irrational beliefs relate to psychological distress in athletes. Psychology of Sport and Exercise, 44, 9-16

Turner, M., \& Barker, J. B. (2013). Examining the efficacy of rational-emotive behavior therapy (REBT) on irrational beliefs and anxiety in elite youth cricketers. Journal of Applied Sport Psychology, 25(1), 131-147

Turner, M., \& Moore, M. (2016). Irrational beliefs predict increased emotional and physical exhaustion in Gaelic football athletes. International Journal of Sport Psychology, 47(2), 187-201

Turner, M. J., Slater, M. J., Dixon, J., \& Miller, A. (2018a). Test-retest reliability of the irrational performance beliefs inventory. European Journal of Sport Science, 18(1), 123-129. https://doi.org/ 10.1080/17461391.2017.1411527.

Turner, M. J., Allen, M., Slater, M. J., Barker, J. B., Woodcock, C., Harwood, C. G., \& McFadyen, K. (2018b). The development and initial validation of the irrational performance beliefs inventory (iPBI). European Journal of Psychological Assessment, 34, 174-180

Turner, M. J., \& Barker, J. B. (2014). Using rational emotive behavior therapy with athletes. The Sport Psychologist, 28(1), 75-90

Turner, M. J., Carrington, S., \& Miller, A. (2019b). Psychological distress across sport participation groups: The mediating effects of secondary irrational beliefs on the relationship between primary irrational beliefs and symptoms of anxiety, anger, and depression. Journal of Clinical Sport Psychology, 13(1), 17-40

Urfa, O., \& Asci, F. H. (2018). Examination of psychometric properties of the irrational performance belief inventory-2. Studies in psychology -psikoloji Calismalar Dergisi, 38(2), 219-236

Wood, A. G., Turner, M. J., Barker, J. B., \& Higgins, S. J. (2017). Investigating the effects of irrational and rational self-statements on motor-skill and hazard-perception performance. Sport, Exercise, and Performance Psychology, 6(4), 384

Ziegler, M., \& Horstmann, K. (2015). Discovering the second side of the coin. Göttingen: Hogrefe Publishing.

Publisher's Note Springer Nature remains neutral with regard to jurisdictional claims in published maps and institutional affiliations. 